\title{
Evaluation of Volatile Compounds Isolated from Pork Loin (Longissimus dorsi) as Affected by Fiber Type of Solid-phase Microextraction (SPME), Preheating and Storage Time
}

\author{
Sung Yong Park ${ }^{1}$, Young Mo Yoon ${ }^{2}$, M. Wes Schilling ${ }^{2}$, and Koo Bok Chin ${ }^{1 *}$ \\ ${ }^{I}$ Department of Animal Science and Biotechnology Research Institute, Chonnam National University, \\ Gwangju 500-757, Korea \\ ${ }^{2}$ Department of Food Science, Nutrition, and Health Promotion, Mississippi State University, \\ Mississippi State, MS, 39762, USA
}

\begin{abstract}
This study was conducted to investigate the effects of heating, fiber type used in solid-phase microextraction (SPME, two phase vs three phase) and storage time on the volatile compounds of porcine M. longissimus dorsi (LD). Volatile compounds were measured using a gas chromatography and mass spectrometry (GC/MS) with a quadrupole mass analyzer. Among the volatile compounds identified, aldehydes (49.33\%), alcohols $(24.63 \%)$ and ketones $(9.85 \%)$ were higher in pre-heated loins $\left(100^{\circ} \mathrm{C} / 30 \mathrm{~min}\right)$, whereas, alcohols $(34.33 \%)$, hydrocarbons $(22.84 \%)$ and ketones $(16.88 \%)$ were higher in non-heated loins. Heating of loins induced the formation of various volatile compounds such as aldehydes (hexanal) and alcohols. The total contents of hydrocarbons, alcohols, and carboxylic acids were higher in two phase fibers, whereas those of esters tended to be higher in three-phase fibers $(p<0.05)$. Most volatile compounds increased $(p<0.05)$ with increased storage time. Thus, the analysis of volatile compounds were affected by the fiber type, while heating and refrigerated storage of pork $\mathrm{M}$. longissimus dorsi increased the volatile compounds derived from lipid oxidation and amino acid catabolism, respectively.
\end{abstract}

Key words : volatile compounds, SPME fiber type, heating, storage time, pork loin

\section{Introduction}

Volatile flavor compounds are important factors affecting palatability of meat and meat products. Various methodologies such as vacuum distillation (VD), static headspace (SH), purge and trap (P\&T), simultaneous distillation/extraction (SDE) and solid-phase microextraction (SPME) have been used to determine the volatile composition of food systems. Among them, the SPME technique was recently developed and has some advantages compared to the other methods. For example, in $\mathrm{SH}$ and SDE method, the heating step can lead to the formation of artifacts. Moreover, SH, SDE and VD methods normally use various solvents for extraction of flavor compounds and contain concentration procedures, which can lead to the loss of low molecular weight volatile compounds. However, SPME is based on absorption, is sol-

*Corresponding author : Koo Bok Chin, Department of Animal Science, Chonnam National University, Gwangju 500-757, Korea, Tel: 82-62-530-2121, Fax: 82-62-530-2129, E-mail: kbchin@chonnam.ac.kr vent free, does not require heating, and has a short analysis time. Analysis of volatile compounds using the SPME technique depends on various factors including fiber type, pressure, absorption time and temperature.

Brunton et al. (2001) evaluated the effects of temperature and pressure on the volatile compound composition of turkey breast using a $75 \mu \mathrm{m}$ Carboxen-polydimethylsiloxane (Carboxen-PDMS) SPME fiber and reported that total volatiles were increased with increased sample temperature, especially, in compounds with low volatility. They also reported that reduced pressure resulted in a 470 fold increase in the recovery of aldehyde and alcohol headspace components, when compared to the levels detected at atmospheric pressure. Ruiz et al. (1998) tested the effects of extraction temperature $\left(40\right.$ and $\left.60^{\circ} \mathrm{C}\right)$ and extraction time (20,40 and $60 \mathrm{~min})$ on the volatile composition of dry-cured ham using a $100 \mu \mathrm{m}$ PDMS fiber, and reported that most volatile compounds had the highest concentration in samples that were heated at $60^{\circ} \mathrm{C}$ for $60 \mathrm{~min}$. Stephen Elmore et al. (2000) compared $75 \mu \mathrm{m}$ Carboxen-PDMS and 50/30 $\mu \mathrm{m}$ divinylbenzene (DVB)Carboxen fibers for their ability to extract volatile com- 
pounds in cooked pork, and reported that the twenty-three compounds that were quantified on both fiber types were significantly different and only two compounds were found at higher levels on the DVB-Carboxen than the Carboxen-PDMS fiber.

Thus, the objective of this study was to investigate the effect of heating, SPME fiber type, and storage time on the volatile profile of porcine M. longissimus dorsi by SPME technique to provide the information for alteration of its flavor characteristic by heating, to investigate suitable fiber type for further analysis, and to monitor variation of volatile composition during refrigerated storage at $4^{\circ} \mathrm{C}$, respectively.

\section{Materials and Methods}

\section{Materials}

Fresh pork loins (M. longissimus dorsi, LD) were purchased from a local meat market (Starkville, MS, USA) and trimmed to remove excess fat and connective tissue. Two SPME fibers $(75 \mu \mathrm{m}$, Carboxen-polydimethylsiloxane, and 50/30 $\mu \mathrm{m}$, divinylbenzene-Carboxen-polydimethyl siloxane) and chlorobenzene were obtained from Supelco (Bellefonte, PA, USA). To investigate the effect of storage time, approximately $100 \mathrm{~g}$ of LD muscle was separated for each treatment, individually vacuum packaged (Model HVT-30, Hollymatic Corp., Countryside, IL) in high performance bags (Vacuum Pouches, KOCH Supplies Inc., Kansas City, MO) and subsequent analyses were performed using $75 \mu \mathrm{m}$ Carboxen-polydimethylsiloxane fiver during storage at $4^{\circ} \mathrm{C}$ for storage times of 0 , 3,6 and $9 \mathrm{~d}$.

\section{Solid Phase Microextraction (SPME)}

Prior to sampling, new fibers were conditioned in a split/splitless GC injector port for $30 \mathrm{~min}$ at $250^{\circ} \mathrm{C}$ to remove any possible contaminants from the fiber coating. This was followed by desorption in the GC injector port for $5 \mathrm{~min}$, with the GC programmed to determine the presence of any extraneous peaks. Approximately $100 \mathrm{~g}$ of LD muscle was homogenized using a food mixer (Model HC306, Black and Decker, Corporation, Towson, $\mathrm{MD}$ ), and $10 \mathrm{~g}$ of homogenate was transferred into a 40 $\mathrm{mL}$ amber glass vial (O.D. $28 \times 98 \mathrm{~mm}$ height, Supelco, Bellefonte, USA) with an open center propylene screw cap and Teflon faced silicone septum (O.D. $22 \mathrm{~mm}$ diameter, Supelco, Bellefonte, USA). Internal standard ( $1 \mu \mathrm{L}$, $200 \mathrm{ppm}$, chlorobenzene) was added into the vial for quantification of the detected volatile compounds. Sam- ples were equilibrated at $50^{\circ} \mathrm{C}$ for $30 \mathrm{~min}$ in the amber glass vials. The SPME fiber was then exposed to the generated sample headspace for $1 \mathrm{hr}$ at $50^{\circ} \mathrm{C}$ in a thermostatic heating block (Reacti-therm Heating/Stirring Module, Pierce Biotechnology Inc., Rockford, USA). For the heat treatment, the samples were heated in the vials at $100^{\circ} \mathrm{C}$ for $30 \mathrm{~min}$ and cooled at room temperature for $10 \mathrm{~min}$ prior to the equilibration and extraction procedures listed above. After extraction, SPME fibers were injected into a splitless injection port of a 5890 Series II gas chromatograph-mass spectrometer (Hewlett-Packard Co., Palo Alto, USA).

\section{Gas Chromatography-Mass Selective Detector (GC-MSD)}

The gas chromatography-mass spectrometer (GC-MS) consisted of an HP 5890 Series II GC/HP 5972 Quadrupole mass selective detector (MSD, Hewlett-Packard Co., California, USA) equipped with an $\mathrm{Rtx}^{\circledR}-5$ (Crossbond ${ }^{\circledR}$ 5\% diphenyl-95\% dimethyl polysiloxane) capillary column (RESTEK, Bellefonte, PA, USA) with the following dimensions: $30 \mathrm{~m}$ length $\times 0.25 \mathrm{~mm}$ i.d. $\times 0.25 \mu \mathrm{m}$ film thickness $\left(\mathrm{d}_{\mathrm{f}}\right)$. GC conditions were as follows: injection port, $250^{\circ} \mathrm{C}$; the oven temperature was programmed at $40^{\circ} \mathrm{C}$ for 5 min with a $8^{\circ} \mathrm{C} / \mathrm{min}$ ramp rate until it reached at $250^{\circ} \mathrm{C}$ where it was held for $5 \mathrm{~min}$ (total running time of $36.25 \mathrm{~min}$ ). MSD conditions were as follows: interface temperature, $250^{\circ} \mathrm{C}$; ionization energy, $70 \mathrm{eV}$; mass range, 33-350 a.m.u.; scan rate, 2.0 scans/s. Ultra high purity helium (Airgas, Columbus, MS) was used as carrier gas at a constant flow rate of $0.96 \mathrm{~mL} / \mathrm{min}$. Volatile compounds were tentatively identified using the Wiley $138 \mathrm{~K}$ Mass Spectral Database (John Wiley and Sons Inc., New York, NY, USA). Relative abundance (ppm) of detected volatile compounds was calculated using the internal standard.

\section{Statistical Analysis}

Three replications of a two factor experiment within a completely randomized design was used to determine the effects $(p<0.05)$ of SPME fiber type (two- and threephase) and heating (heat and non-heat) on the volatile composition of porcine LD muscle. Data were analyzed using a two-way analysis of variance (ANOVA) in SPSS 12.0 software for windows. The data related to storage time were analyzed using one-way ANOVA in the same program. Means were separated $(p<0.05)$ by the Duncan's multiple range test. 


\section{Results and Discussion}

\section{Effect of Heating and Fiber Type on Volatile Com- pounds}

Volatile compounds tentatively identified were classified by chemical groups and listed in Tables 1-4. One hundred eleven volatile compounds were identified in pre-heated (94) and non-heated (82) loins, including hydrocarbons (25), alcohols (32), aldehydes (12), ketones (12), esters (11), carboxylic acids (4), nitrogen compounds (4), sulfur compounds (7) and furans (4). Among volatile compounds identified in pre-heated loins, aldehydes $(225.85 \mu \mathrm{g} / \mathrm{kg})$ had the highest concentration, fol- lowed by alcohols $(112.77 \mu \mathrm{g} / \mathrm{kg})$, ketones $(45.11 \mu \mathrm{g} / \mathrm{kg})$ and hydrocarbons $(38.94 \mu \mathrm{g} / \mathrm{kg})$ and, whereas alcohols $(63.79 \mu \mathrm{g} / \mathrm{kg})$, hydrocarbons $(42.43 \mu \mathrm{g} / \mathrm{kg})$ and ketones $(31.37 \mu \mathrm{g} / \mathrm{kg})$ were the predominant compounds in nonheated loins. Also, 2-ethyl-1-hexanol $(42.56 \mu \mathrm{g} / \mathrm{kg})$ and hexanal $(157.17 \mu \mathrm{g} / \mathrm{kg})$ were the most representative compounds in non-heated and pre-heated loins, respectively (Tables 2 and 3). Estevez et al. (2003) reported that 2-ethyl-1-hexanol was the major compound in raw loins, and hexanal was the major volatile compound in cooked loins, generated during cooking at $80^{\circ} \mathrm{C}$ for 10 min using SPME-GCMS, which supports the results of this study. The concentrations of total volatile compounds in pre-

Table 1. Effects of heating and SPME fiber type on the concentration of hydrocarbons released from pork M. longissimus dorsi

\begin{tabular}{|c|c|c|c|c|c|}
\hline \multirow{2}{*}{$\begin{array}{l}\text { Tentatively identified } \\
\text { volatile compounds }\end{array}$} & \multirow{2}{*}{ Interaction } & \multicolumn{2}{|c|}{ Heating } & \multicolumn{2}{|c|}{ Fiber type } \\
\hline & & cooked & uncooked & 2 phase & 3 phase \\
\hline \multicolumn{6}{|l|}{ Hydrocarbons } \\
\hline \multicolumn{6}{|l|}{ Aliphatic hydrocarbons } \\
\hline 3-octene & No & 1.86 & 1.87 & $3.19^{\mathrm{a}}$ & $0.54^{\mathrm{b}}$ \\
\hline 3-octene, $(\mathrm{Z})$ & No & $t^{1) b}$ & $2.63^{\mathrm{a}}$ & 1.50 & 1.13 \\
\hline 4-nonene & No & $5.47^{\mathrm{a}}$ & $0.31^{\mathrm{b}}$ & 2.92 & 2.86 \\
\hline 1,3,5-cycloheptatriene & Yes & $0.76^{\mathrm{a}}$ & $\mathrm{t}^{\mathrm{b}}$ & $\mathrm{t}^{\mathrm{b}}$ & $0.76^{\mathrm{a}}$ \\
\hline undecane & Yes & $4.34^{\mathrm{b}}$ & $15.94^{\mathrm{a}}$ & $17.22^{\mathrm{a}}$ & $3.06^{\mathrm{b}}$ \\
\hline tetradecane & No & 2.58 & 2.76 & $2.07^{\mathrm{b}}$ & $3.27^{\mathrm{a}}$ \\
\hline pentadecane & No & 0.82 & 0.41 & $t^{b}$ & $1.23^{\mathrm{a}}$ \\
\hline hexadecane & No & 1.31 & 1.05 & $0.93^{\mathrm{b}}$ & $1.43^{\mathrm{a}}$ \\
\hline nonadecane & No & 0.16 & 0.17 & $\mathrm{t}^{\mathrm{b}}$ & $0.33^{\mathrm{a}}$ \\
\hline Total & & $17.29^{b}$ & $25.14^{\mathrm{a}}$ & $27.82^{\mathrm{a}}$ & $14.61^{\mathrm{b}}$ \\
\hline \multicolumn{6}{|l|}{ Branched hydrocarbons } \\
\hline 2-methyl-butane & Yes & $8.21^{\mathrm{a}}$ & $3.03^{\mathrm{b}}$ & $8.75^{\mathrm{a}}$ & $2.49^{\mathrm{b}}$ \\
\hline 3-ethyl-2-methyl-pentane & No & 1.89 & 1.85 & 2.08 & 1.67 \\
\hline 2,2,3-trimethyl-hexane & No & 1.42 & 0.86 & 1.57 & 0.71 \\
\hline 3,7-dimethyl-1-octene & No & $\mathrm{t}^{\mathrm{b}}$ & $1.89^{\mathrm{a}}$ & 1.07 & 0.82 \\
\hline ethyl-cyclobutane & No & 6.94 & 4.96 & 4.80 & 7.10 \\
\hline 2,2-dimethyl-octane & No & 1.89 & 1.72 & 1.65 & 1.97 \\
\hline methyl-cyclopentane & No & $0.45^{\mathrm{a}}$ & $\mathrm{t}^{\mathrm{b}}$ & 0.23 & 0.23 \\
\hline 4-methyl-decane & Yes & $\mathrm{t}^{\mathrm{b}}$ & $0.94^{\mathrm{a}}$ & $0.37^{\mathrm{b}}$ & $0.57^{\mathrm{a}}$ \\
\hline 2-cyclohexyl-dodecane & No & 0.08 & 0.10 & $t^{b}$ & $0.18^{\mathrm{a}}$ \\
\hline 6-methyl-octadecane & No & $0.37^{\mathrm{a}}$ & $0.18^{\mathrm{b}}$ & $0.18^{\mathrm{b}}$ & $0.37^{\mathrm{a}}$ \\
\hline Total & & 21.27 & 15.53 & 20.70 & 16.10 \\
\hline \multicolumn{6}{|l|}{ Aromatic hydrocarbons } \\
\hline 1-ethyl-4-methyl-benzene & Yes & $\mathrm{t}^{\mathrm{b}}$ & $1.11^{\mathrm{a}}$ & $0.33^{\mathrm{b}}$ & $0.77^{\mathrm{a}}$ \\
\hline 1,2,3-trimethyl-benzene & No & $\mathrm{t}^{\mathrm{b}}$ & $0.31^{\mathrm{a}}$ & 0.10 & 0.21 \\
\hline 1-methyl-3-propyl-benzene & No & 0.22 & 0.22 & $\mathrm{t}^{\mathrm{b}}$ & $0.44^{\mathrm{a}}$ \\
\hline $1,2,4,5$-tetramethyl-benzene & No & 0.14 & $\mathrm{t}$ & $\mathrm{t}$ & 0.14 \\
\hline (1-butyloctyl)-benzene & No & 0.02 & 0.06 & $0.08^{\mathrm{a}}$ & $\mathrm{t}^{\mathrm{b}}$ \\
\hline (1-propylnonyl)-benzene & Yes & $\mathrm{t}^{\mathrm{b}}$ & $0.07^{\mathrm{a}}$ & $0.07^{\mathrm{a}}$ & $\mathrm{t}^{\mathrm{b}}$ \\
\hline Total & & $0.38^{\mathrm{b}}$ & $1.76^{\mathrm{a}}$ & $0.58^{\mathrm{b}}$ & $1.56^{\mathrm{a}}$ \\
\hline Total hydrocarbons & & 38.94 & 42.43 & $49.10^{\mathrm{a}}$ & $32.27^{\mathrm{b}}$ \\
\hline
\end{tabular}

${ }_{\mathrm{a}, \mathrm{b}}$ Means with a same superscript within a same row are not different $(p>0.05)$.

${ }^{1)} \mathrm{t}=$ trace amount. 
Table 2. Effects of heating and SPME fiber type on the concentration of alcohols released from pork M. longissimus dorsi

\begin{tabular}{|c|c|c|c|c|c|}
\hline \multirow{2}{*}{$\begin{array}{l}\text { Tentatively identified } \\
\text { volatile compounds }\end{array}$} & \multirow{2}{*}{ Interaction } & \multicolumn{2}{|c|}{ Heating } & \multicolumn{2}{|c|}{ Fiber type } \\
\hline & & cooked & uncooked & 2 phase & 3 phase \\
\hline \multicolumn{6}{|l|}{ Alcohols } \\
\hline \multicolumn{6}{|l|}{ Aliphatic alcohols } \\
\hline 2-buten-1-ol & Yes & $1.53^{\mathrm{a}}$ & $t^{1) b}$ & $1.53^{\mathrm{a}}$ & $t^{b}$ \\
\hline 1-butanol & No & 3.51 & 2.42 & $4.60^{\mathrm{a}}$ & $1.33^{\mathrm{b}}$ \\
\hline 1-penten-3-ol & No & $0.64^{\mathrm{a}}$ & $t^{b}$ & 0.42 & 0.23 \\
\hline 3-heptanol & No & $t^{b}$ & $0.63^{\mathrm{a}}$ & 0.34 & 0.29 \\
\hline 1-octen-3-ol & No & $13.28^{\mathrm{a}}$ & $2.23^{\mathrm{b}}$ & 6.00 & 9.52 \\
\hline 2-octen-1-ol & No & $1.51^{\mathrm{a}}$ & $t^{b}$ & 0.71 & 0.80 \\
\hline 2-nonen-1-ol & No & $25.31^{\mathrm{a}}$ & $3.41^{\mathrm{b}}$ & 11.39 & 17.32 \\
\hline 2-decen-1-ol & No & $2.11^{\mathrm{a}}$ & $0.72^{\mathrm{b}}$ & $0.58^{\mathrm{b}}$ & $2.25^{\mathrm{a}}$ \\
\hline 2-undecen-1-ol & No & 0.50 & 0.42 & $0.29^{b}$ & $0.63^{\mathrm{a}}$ \\
\hline 2-tridecen-1-ol & Yes & $0.89^{\mathrm{a}}$ & $0.38^{\mathrm{b}}$ & $0.21^{\mathrm{b}}$ & $1.06^{\mathrm{a}}$ \\
\hline cyclododecanol & No & $0.43^{\mathrm{a}}$ & $t^{b}$ & 0.10 & 0.32 \\
\hline 10-pentadecenol & Yes & $0.42^{\mathrm{a}}$ & $t^{b}$ & $0.11^{\mathrm{b}}$ & $0.32^{\mathrm{a}}$ \\
\hline Total & & $50.12^{\mathrm{a}}$ & $10.20^{\mathrm{b}}$ & 26.27 & 34.05 \\
\hline \multicolumn{6}{|l|}{ Branched alcohols } \\
\hline 2-methyl-1-propanol & No & $6.86^{\mathrm{a}}$ & $t^{b}$ & 4.89 & 1.97 \\
\hline 3-methyl-3-buten-1-ol & No & 1.44 & 1.49 & 1.90 & 1.03 \\
\hline 2-ethyl-1-pentanol & No & 2.55 & 1.04 & $3.15^{\mathrm{a}}$ & $0.45^{\mathrm{b}}$ \\
\hline 3-methyl-3-cyclohexen-1-ol & No & $0.50^{\mathrm{a}}$ & $t^{b}$ & 0.16 & 0.34 \\
\hline 3-methyl-1,5-pentanediol & No & $18.26^{\mathrm{a}}$ & $t^{b}$ & $18.08^{\mathrm{a}}$ & $0.18^{\mathrm{b}}$ \\
\hline 2-butoxy-ethanol & No & 2.19 & 1.20 & $3.39^{\mathrm{a}}$ & $t^{b}$ \\
\hline 3,4-dimethyl-3-hexanol & No & 0.43 & 0.57 & 0.53 & 0.47 \\
\hline 1-butoxy-2-propanol & No & 0.71 & 0.70 & 0.74 & 0.67 \\
\hline 2-ethyl-4-methyl-1-pentanol & No & $\mathrm{t}^{\mathrm{b}}$ & $0.40^{\mathrm{a}}$ & 0.16 & 0.24 \\
\hline 4-ethylcyclohexanol & No & $2.25^{\mathrm{a}}$ & $t^{b}$ & 1.40 & 0.85 \\
\hline 2-ethyl-1-hexanol & Yes & $18.65^{\mathrm{b}}$ & $42.56^{\mathrm{a}}$ & $44.90^{\mathrm{a}}$ & $16.31^{\mathrm{b}}$ \\
\hline 4-ethyl-1-octyn-3-ol & Yes & $0.51^{\mathrm{a}}$ & $t^{b}$ & $t^{b}$ & $0.51^{\mathrm{a}}$ \\
\hline 3,7-dimethyl-1,7-octanediol & No & $5.14^{\mathrm{a}}$ & $2.77^{\mathrm{b}}$ & $3.04^{\mathrm{b}}$ & $4.87^{\mathrm{a}}$ \\
\hline 2-butyl-1-octanol & Yes & 0.23 & 0.27 & 0.09 & 0.41 \\
\hline 2-methyl-1-undecanol & No & 0.27 & 0.39 & $t^{b}$ & $0.66^{\mathrm{a}}$ \\
\hline 2-hexyl-1-octanol & No & 0.26 & 0.10 & $t^{b}$ & $0.35^{\mathrm{a}}$ \\
\hline 2-methyl-1-hexadecanol & No & 0.03 & 0.03 & $t^{b}$ & $0.05^{\mathrm{a}}$ \\
\hline Total & & 60.26 & 51.52 & $82.42^{\mathrm{a}}$ & $29.35^{\mathrm{b}}$ \\
\hline \multicolumn{6}{|l|}{ Aromatic alcohols } \\
\hline 3-phenoxy-1-propanol & Yes & $0.72^{\mathrm{a}}$ & $\mathrm{t}^{\mathrm{b}}$ & $t^{b}$ & $0.72^{\mathrm{a}}$ \\
\hline 2-methyl-5-(1-methylethyl)-phenol & No & 0.62 & 0.82 & $1.44^{\mathrm{a}}$ & $t^{b}$ \\
\hline 5-methyl-2-(1-methylethyl)-phenol & No & 1.05 & 1.25 & $2.30^{\mathrm{a}}$ & $t^{b}$ \\
\hline Total & & 2.39 & 2.07 & $3.74^{\mathrm{a}}$ & $0.72^{b}$ \\
\hline Total alcohols & & $112.77^{\mathrm{a}}$ & $63.79^{\mathrm{b}}$ & $112.44^{\mathrm{a}}$ & $64.12^{\mathrm{b}}$ \\
\hline
\end{tabular}

${ }_{\mathrm{a}, \mathrm{b}}$ Means with a same superscript within a same row are not different $(p>0.05)$.

${ }^{1)} \mathrm{t}=$ trace amount.

heated and non-heated loins were approximately 457.9 and $185.8 \mu \mathrm{g} / \mathrm{kg}$. Poligne et al. (2002) studied the effects of cooking on volatile compound formation in pork and reported that the total chromatogram peak area for cooked meat was 2.4-fold higher than that of raw meat, which was similar to the results from this study. Ahn et al. (1999) evaluated the volatile profiles of raw and cooked turkey meat and reported that total volatiles were increased with cooking, which was also in agreement with the results from this study. The flavor profiles in cooked meat are performed as a result of thermally induced reactions such as the Maillard reaction and the 
Table 3. Effects of heating and SPME fiber type on the concentration of aldehydes and ketones released from pork M. longissimus dorsi

\begin{tabular}{|c|c|c|c|c|c|}
\hline \multirow{2}{*}{$\begin{array}{l}\text { Tentatively identified } \\
\text { volatile compounds }\end{array}$} & \multirow{2}{*}{ Interaction } & \multicolumn{2}{|c|}{ Heating } & \multicolumn{2}{|c|}{ Fiber type } \\
\hline & & cooked & uncooked & 2 phase & 3 phase \\
\hline \multicolumn{6}{|l|}{ Aldehydes } \\
\hline \multicolumn{6}{|l|}{ Aliphatic aldehydes } \\
\hline pentanal & No & $29.81^{\mathrm{a}}$ & $4.82^{\mathrm{b}}$ & 23.26 & 11.36 \\
\hline hexanal & No & $157.17^{\mathrm{a}}$ & $0.81^{\mathrm{b}}$ & 86.78 & 71.20 \\
\hline heptanal & No & $11.87^{\mathrm{a}}$ & $t^{1) b}$ & 6.67 & 5.20 \\
\hline 2-heptenal, (Z) & No & $1.68^{\mathrm{a}}$ & $\mathrm{t}^{\mathrm{b}}$ & 0.83 & 0.85 \\
\hline octanal & No & $17.25^{\mathrm{a}}$ & $t^{b}$ & 8.13 & 9.13 \\
\hline 2-octenal & No & $1.91^{\mathrm{a}}$ & $0.47^{\mathrm{b}}$ & $0.95^{\mathrm{b}}$ & $1.43^{\mathrm{a}}$ \\
\hline 2,4-dodecadienal & No & 0.15 & 0.32 & 0.09 & 0.39 \\
\hline pentadecanal & No & $0.75^{\mathrm{a}}$ & $t^{b}$ & 0.26 & 0.49 \\
\hline Total & & $220.58^{a}$ & $6.42^{\mathrm{b}}$ & 126.96 & 100.04 \\
\hline \multicolumn{6}{|l|}{ Branched aldehydes } \\
\hline 3-methyl-butanal & No & $2.72^{\mathrm{a}}$ & $0.51^{\mathrm{b}}$ & $2.49^{\mathrm{a}}$ & $0.74^{\mathrm{b}}$ \\
\hline 3-methyl-hexanal & No & 1.04 & 1.03 & 1.22 & 0.85 \\
\hline 4-methyl-hexanal & No & 0.96 & $\mathrm{t}$ & 0.96 & $\mathrm{t}$ \\
\hline Total & & $4.71^{\mathrm{a}}$ & $1.54^{\mathrm{b}}$ & $4.67^{\mathrm{a}}$ & $1.58^{\mathrm{b}}$ \\
\hline \multicolumn{6}{|l|}{ Aromatic aldehydes } \\
\hline 4-(1-methylethyl)-benzaldehyde & No & 0.55 & 0.58 & $1.12^{\mathrm{a}}$ & $t^{b}$ \\
\hline Total aldehydes & & $225.85^{\mathrm{a}}$ & $8.54^{\mathrm{b}}$ & 132.75 & 101.63 \\
\hline \multicolumn{6}{|l|}{ Ketones } \\
\hline \multicolumn{6}{|l|}{ Aliphatic ketones } \\
\hline 2-pentanone & No & 1.78 & 1.70 & $2.45^{\mathrm{a}}$ & $1.04^{\mathrm{b}}$ \\
\hline 2,3-octanedione & No & $9.47^{\mathrm{a}}$ & $1.68^{\mathrm{b}}$ & 4.82 & 6.33 \\
\hline 9-heptadecanone & No & 0.15 & $\mathrm{t}$ & $\mathrm{t}$ & 0.15 \\
\hline Total & & $11.40^{\mathrm{a}}$ & $3.38^{\mathrm{b}}$ & 7.27 & 7.52 \\
\hline \multicolumn{6}{|l|}{ Branched ketones } \\
\hline 1-hydroxy-2-propanone & Yes & $t^{b}$ & $4.19^{\mathrm{a}}$ & $4.19^{\mathrm{a}}$ & $t^{b}$ \\
\hline 3-hydroxy-2-butanone & No & 9.41 & 15.80 & $18.27^{\mathrm{a}}$ & $6.94^{\mathrm{b}}$ \\
\hline 4-methyl-2-hexanone & No & $3.88^{\mathrm{a}}$ & $2.79^{\mathrm{b}}$ & 3.11 & 3.57 \\
\hline 2-methyl-3-octanone & No & $6.30^{\mathrm{a}}$ & $2.25^{\mathrm{b}}$ & 4.81 & 3.73 \\
\hline 3,5,5-trimethyl-2-cyclohexen-1-one & No & $0.43^{\mathrm{b}}$ & $1.09^{\mathrm{a}}$ & $0.28^{\mathrm{b}}$ & $1.24^{\mathrm{a}}$ \\
\hline 3,6-dimethyl-octan-2-one & Yes & $0.56^{\mathrm{a}}$ & $0.22^{\mathrm{b}}$ & $t^{b}$ & $0.78^{\mathrm{a}}$ \\
\hline Total & & 20.58 & 26.33 & $30.66^{\mathrm{a}}$ & $16.25^{\mathrm{b}}$ \\
\hline \multicolumn{6}{|l|}{ Aromatic ketones } \\
\hline 2,2-dihydroxy-1-phenyl-ethanone & No & $11.32^{\mathrm{a}}$ & $t^{b}$ & 5.75 & 5.58 \\
\hline 1-phenyl-1-butanone & No & 1.47 & 1.27 & $0.83^{\mathrm{b}}$ & $1.91^{\mathrm{a}}$ \\
\hline benzophenone & No & 0.33 & 0.38 & 0.35 & 0.36 \\
\hline Total & & $13.13^{\mathrm{a}}$ & $1.65^{\mathrm{b}}$ & 6.93 & 7.85 \\
\hline Total ketones & & 45.11 & 31.37 & 44.85 & 31.62 \\
\hline
\end{tabular}

${ }_{\mathrm{a}, \mathrm{b}}$ Means with a same superscript within a same row are not different $(p>0.05) .{ }^{1)} \mathrm{t}=$ trace amount.

degradation of lipids during heating (Mottram, 1998). In addition, these thermally induced reactions contribute to the formation of aldehydes and alcohols.

Volatile compounds extracted from pork loins were also affected $(p<0.05)$ by fiber type. Ninety-one and 99 volatile compounds were identified in two- and three-phase fibers, respectively. The two-phase fiber (Carboxen-PDMS) was effective at extracting aroma compounds with low- boiling points in the molecular mass range of 30-225, whereas the three-phase fiber (DVB-Carboxen on PDMS), which is comprised of a layer of PDMS-DVB over a layer of Carboxen-PDMS, was effective at extracting higher boiling point compounds in the molecular mass range of 40-275. These results are similar to those of other researchers who reported that PDMS-DVB addition to Carboxen PDMS allowed for the absorbtion of com- 
pounds with a wide range of molecular weights (Machiels and Istasse, 2003; Stephen Elmore et al., 2000). Gianelli et al. (2002) also reported that DVB-Carboxen on a PDMS fiber extracted a higher number of volatile compounds than a Carboxen-PDMS fiber (60 vs 51, respectively) in dry-cured ham, which is in agreement with our results. Aldehydes, alcohols, hydrocarbons and ketones were the main compounds identified and were extracted with concentrations of $132.75,112.44,49.10$ and 44.85 $\mu \mathrm{g} / \mathrm{kg}$, respectively, using a two-phase fiber. These chemical groups were also the predominant compounds extracted with the three phase fibers with concentrations of $101.63,64.12,32.27$ and $31.62 \mu \mathrm{g} / \mathrm{kg}$, respectively. Total concentrations of volatile compounds extracted from twoand three-phase fibers were 381.9 and $261.8 \mu \mathrm{g} / \mathrm{kg}$. Thus, two-phase fibers extracted more volatile compounds than the three-phase fibers in most chemical groups. These results are in agreement with those who reported in the previous studies of cooked pork and beef, and dry cured ham (Garcia-Esteban et al., 2004; Gianelli et al., 2002; Machiels and Istasse, 2003; Stephen Elmore et al., 2000).

\section{Hydrocarbons}

Pre-heating increased $(p<0.05)$ the formation of aliphatic (2) and branched (3) hydrocarbons such as 4-nonene, 1,3,5-cycloheptatriene, 2-methyl-butane, methyl-cyclopentane and 6-methyl-octadecane, and decreased $(p<$ $0.05)$ the concentrations of hydrocarbons such as (Z)-3octene, undecane, 3,7-dimethyl-1-octene, 4-methyl-decane, 1-ethyl-4-methyl-benzene, 1,2,3-trimethyl-benzene and (1propylnonyl)-beznene (Table 1). These results are similar to those of previous studies showing that total aliphatic hydrocarbons decreased in pork bellies after cooking (Estevez et al., 2003; Poligne et al., 2002). Three phase fibers extracted a higher number of hydrocarbons than the two-phase fibers, but the two phase fibers $(49.10 \mu \mathrm{g} / \mathrm{kg})$ extracted a higher concentration $(p<0.05)$ of hydrocarbons than the three phase fibers $(32.27 \mu \mathrm{g} / \mathrm{kg})$. These results were supported by a previous study of Stephen Elmore et al. (2000) and Garcia-Esteban et al. (2004) who studied identical fibers to the ones used in this study, and analyzed cooked pork meat and dry cured ham, respectively.

\section{Alcohols}

Most alcohols were detected at higher $(p<0.05)$ concentrations in pre-heated loins than non-heated counterpart, especially, 1-octen-3-ol, 2-nonen-1-ol and 3-methyl-1,5pentanediol (Table 2). However, most representative 2- ethyl-1-hexanol in non-heated loins was decreased by heating $(p<0.05)$. Increases in alcohols with pre-heating may be derived from lipid oxidation because heating accelerates lipid oxidation and inactivates enzyme for amino acid catabolism, whereas, 2-ethyl-1-hexanol may not be generated from lipid oxidation since its concentration decreases when the sample is heated. Differences $(p<0.05)$ in total aliphatic alcohols and total alcohols were found between pre-heated and non-heated loins; these results are in agreement with previous studies (Ahn et al., 1999; Poligne et al., 2002). Eighteen compounds including aliphatic (6), branched (9) and aromatic (3) alcohols were affected $(p<0.05)$ by SPME fiber type. Among these compounds, branched alcohols (especially 3-methyl-1,5-pentanediol and 2-ethyl-1-hexanol) had higher concentrations $(p<0.05)$ when the two phase fiber was used. Moreover, total concentration of alcohols for the two-phase fiber was $112.44 \mu \mathrm{g} / \mathrm{kg}$, approximately 1.8 fold higher than that of the three-phase fiber $(p<0.05)$. These trends were also reported by Stephen Elmore et al. (2000) and Garcia-Esteban et al. (2004), who reported that total concentration and area of alcohols for the Carboxen-PDMS fiber were 3.5- and 1.6-fold higher than those for DVB-Carboxen on a PDMS fiber, respectively.

\section{Aldehydes and Ketones}

Twelve aldehydes were identified in the pork samples (Table 3). Aliphatic aldehydes, especially, pentanal, hexanal, heptanal and octanal increased $(p<0.05)$ with heating. Hexanal has been used as an index to assess the degree of lipid oxidation (Beltran et al., 2003; Morcuende et al., 2003) because it is formed during the oxidation of linoleic acid via the 13-hydroperoxide, has an odor described as "grassy", and contributes to off-flavor formation (SanchesSilva et al., 2004). In addition, it was the most representative compound in heated loins at $157.17 \mu \mathrm{g} / \mathrm{kg}$, approximately 194-fold higher than in non-heated loins. These results are similar to those reported in other studies (Ahn et al., 1999; Estevez et al., 2003; Poligne et al., 2002). However, hexanal concentration is dependent on raw material as well as heating since turkey thigh meat, pork bellies and lean pig loins increased 4.1-, 4.2- and 103.7fold as compared to non-heated samples, respectively. Therefore, pork loins may be the most sensitive to hexanal formation when exposed to heat. Among ketones, concentrations of 2,3-octanedione, 4-methyl-2-hexanone, 2-methyl-3-octanone, 3,6-dimethyl-octan-2-one and 2,2dihydroxy-1-phenyl-ethanone increased $(p<0.05)$ with heat treatment, whereas, 1-hydroxy-2-propanone and 3,5,5-tri- 
Table 4. Effects of heating and SPME fiber type on the concentration of esters, acid, N- and S-containing compounds, and furans released from pork M. longissimus dorsi

\begin{tabular}{|c|c|c|c|c|c|}
\hline \multirow{2}{*}{$\begin{array}{l}\text { Tentatively identified } \\
\text { volatile compounds }\end{array}$} & \multirow{2}{*}{ Interaction } & \multicolumn{2}{|c|}{ Heating } & \multicolumn{2}{|c|}{ Fiber type } \\
\hline & & cooked & uncooked & 2 phase & 3 phase \\
\hline \multicolumn{6}{|l|}{ Esters } \\
\hline \multicolumn{6}{|l|}{ Aliphatic esters } \\
\hline methyl butanoate & No & $t^{1) b}$ & $2.24^{\mathrm{a}}$ & 1.34 & 0.90 \\
\hline methyl hexanoate & No & $0.26^{\mathrm{b}}$ & $0.92^{\mathrm{a}}$ & 0.45 & 0.74 \\
\hline pentyl hexanoate & No & 0.35 & 0.26 & $0.61^{\mathrm{a}}$ & $t^{b}$ \\
\hline heptyl butanoate & No & 1.47 & 1.91 & $1.17^{\mathrm{b}}$ & $2.21^{\mathrm{a}}$ \\
\hline ethyl linolate & No & $\mathrm{t}$ & 0.02 & $\mathrm{t}$ & 0.02 \\
\hline Total & & 2.09 & 5.35 & 3.57 & 3.87 \\
\hline \multicolumn{6}{|l|}{ Branched esters } \\
\hline 3-methylbutyl butanoate & No & 0.99 & 1.04 & $2.03^{\mathrm{a}}$ & $t^{b}$ \\
\hline 3-methyl-1-butyl acetate & No & $t^{b}$ & $2.07^{\mathrm{a}}$ & 0.72 & 1.36 \\
\hline 2-butoxyethyl acetate & No & 2.22 & 1.87 & $t^{b}$ & $4.09^{\mathrm{a}}$ \\
\hline 3-methylheptyl acetate & No & 2.12 & 1.83 & $0.95^{\mathrm{a}}$ & $3.00^{\mathrm{b}}$ \\
\hline 2-ethylhexyl-2-propenoate & Yes & $2.11^{\mathrm{a}}$ & $0.68^{\mathrm{b}}$ & $0.55^{\mathrm{b}}$ & $2.24^{\mathrm{a}}$ \\
\hline 2-ethylhexyl butyrate & No & 0.57 & 0.51 & $t^{b}$ & $1.08^{\mathrm{a}}$ \\
\hline Total & & 8.01 & 8.01 & $4.25^{\mathrm{b}}$ & $11.77^{\mathrm{a}}$ \\
\hline Total esters & & 10.10 & 13.36 & $7.83^{b}$ & $15.63^{\mathrm{a}}$ \\
\hline \multicolumn{6}{|l|}{ Carboxylic acid } \\
\hline butanoic acid & No & $t^{b}$ & $3.74^{\mathrm{a}}$ & 1.74 & 1.99 \\
\hline 2,2-dimethyl-propanoic acid & No & 7.62 & 9.42 & $15.17^{\mathrm{a}}$ & $1.87^{\mathrm{b}}$ \\
\hline 2-methyl-2-ethyl-propanoic acid & No & 0.30 & 0.22 & $t^{b}$ & $0.53^{\mathrm{a}}$ \\
\hline oleic acid & Yes & $0.58^{\mathrm{a}}$ & $t^{b}$ & $0.15^{\mathrm{b}}$ & $0.43^{\mathrm{a}}$ \\
\hline Total carboxylic acid & & 8.51 & 13.38 & $17.07^{\mathrm{a}}$ & $4.82^{b}$ \\
\hline \multicolumn{6}{|l|}{ N-containing compounds } \\
\hline ethylamine & No & 0.03 & 0.07 & $\mathrm{t}^{\mathrm{b}}$ & $0.10^{\mathrm{a}}$ \\
\hline cyclobutylamine & No & $0.26^{\mathrm{a}}$ & $0.07^{\mathrm{b}}$ & 0.22 & 0.11 \\
\hline 4-amino-1-butanol & No & $t^{b}$ & $4.37^{\mathrm{a}}$ & 2.71 & 1.66 \\
\hline pyridine & No & 0.55 & $\mathrm{t}$ & 0.36 & 0.19 \\
\hline Total N-containing compounds & & $0.84^{\mathrm{b}}$ & $4.52^{\mathrm{a}}$ & 3.30 & 2.06 \\
\hline \multicolumn{6}{|l|}{ S-containing compounds } \\
\hline methanethiol & No & $2.39^{\mathrm{a}}$ & $t^{b}$ & 1.30 & 1.10 \\
\hline 1-propanethiol & No & 0.62 & $\mathrm{t}$ & 0.62 & $\mathrm{t}$ \\
\hline acetyl mercaptan & Yes & $t^{b}$ & $5.48^{\mathrm{a}}$ & $4.80^{\mathrm{a}}$ & $0.69^{\mathrm{b}}$ \\
\hline dimethyl disulfide & No & 1.45 & 1.06 & 1.39 & 1.12 \\
\hline 2-methyl-3-pentanethiol & Yes & $0.53^{\mathrm{a}}$ & $t^{b}$ & $\mathrm{t}^{\mathrm{b}}$ & $0.53^{\mathrm{a}}$ \\
\hline dimethyl trisulfide & No & $1.58^{\mathrm{a}}$ & $\mathrm{t}^{\mathrm{b}}$ & 1.08 & 0.50 \\
\hline dimethyl tetrasulfide & No & $0.96^{\mathrm{a}}$ & $t^{b}$ & 0.50 & 0.46 \\
\hline Total S-containing compounds & & 7.54 & 6.54 & 9.69 & 4.40 \\
\hline \multicolumn{6}{|l|}{ Furans } \\
\hline tetrahydro-furan & Yes & $t^{b}$ & $0.21^{\mathrm{a}}$ & $t^{b}$ & $0.21^{\mathrm{a}}$ \\
\hline tetrahydro-2,2,4,4-tetramethyl-furan & Yes & $t^{b}$ & $1.53^{\mathrm{a}}$ & $0.41^{\mathrm{b}}$ & $1.12^{\mathrm{a}}$ \\
\hline 2-pentyl-furan & No & $8.11^{\mathrm{a}}$ & $t^{b}$ & 4.32 & 3.79 \\
\hline dibenzofuran & No & $0.10^{\mathrm{b}}$ & $0.15^{\mathrm{a}}$ & 0.13 & 0.12 \\
\hline Total furans & & $8.21^{\mathrm{a}}$ & $1.90^{\mathrm{b}}$ & 4.86 & 5.25 \\
\hline
\end{tabular}

${ }^{\mathrm{a}, \mathrm{b}}$ Means with a same superscript within a same row are not different $(p>0.05) .{ }^{1)} \mathrm{t}=$ trace amount.

methyl-2-cyclohexen-1-one concentrations decreased ( $p<$ 0.05 ) with heat treatment (Table 3). The two-phase fiber extracted higher concentrations $(p<0.05)$ of 2-pentanone, 1-hydroxy-2-propanone and 3-hydroxy-2-butanone than the three-phase fiber, but extracted less $(p<0.05)$ of 3,5,5trimethyl-2-cyclohexen-1-one, 3,6-dimethyl-octan-2-one and 
Table 5. Volatile compounds released from pork M. longissimus dorsi during storage at $4^{\circ} \mathrm{C}$

$(\mu \mathrm{g} / \mathrm{kg})$

\begin{tabular}{|c|c|c|c|c|}
\hline \multirow{2}{*}{$\begin{array}{l}\text { Tentatively identified } \\
\text { volatile compounds }\end{array}$} & \multicolumn{4}{|c|}{ Storage time (d) } \\
\hline & 0 & 3 & 6 & 9 \\
\hline \multicolumn{5}{|l|}{ Hydrocarbons } \\
\hline \multicolumn{5}{|l|}{ Aliphatic hydrocarbons } \\
\hline 1,3,5-cycloheptatriene & $t^{1) b}$ & $t^{b}$ & $t^{b}$ & $2.50^{\mathrm{a}}$ \\
\hline tetradecane & $2.32^{\mathrm{b}}$ & $2.35^{\mathrm{b}}$ & $5.47^{\mathrm{a}}$ & $2.94^{\mathrm{b}}$ \\
\hline hexadecane & $0.99^{\mathrm{b}}$ & $0.74^{\mathrm{b}}$ & $2.12^{\mathrm{a}}$ & $0.91^{\mathrm{b}}$ \\
\hline \multicolumn{5}{|l|}{ Branched hydrocarbons } \\
\hline 2-methyl-butane & $3.86^{\mathrm{a}}$ & $0.48^{\mathrm{b}}$ & $t^{b}$ & $t^{b}$ \\
\hline ethyl-cyclobutane & $4.32^{\mathrm{ab}}$ & $1.82^{\mathrm{b}}$ & $4.66^{\mathrm{a}}$ & $6.81^{\mathrm{a}}$ \\
\hline 3-ethyl-2,2-dimethyl-pentane & $\mathrm{t}^{\mathrm{c}}$ & $0.45^{\mathrm{b}}$ & $1.59^{\mathrm{a}}$ & $0.62^{b}$ \\
\hline 3,5-dimethyl-octane & $\mathrm{t}^{\mathrm{b}}$ & $0.61^{\mathrm{b}}$ & $2.02^{\mathrm{a}}$ & $0.53^{\mathrm{b}}$ \\
\hline \multicolumn{5}{|l|}{ Aromatic hydrocarbons } \\
\hline 1-methyl-3-propyl-benzene & $\mathrm{t}^{\mathrm{c}}$ & $0.31^{\mathrm{b}}$ & $0.65^{\mathrm{a}}$ & $0.79^{\mathrm{a}}$ \\
\hline \multicolumn{5}{|l|}{ Alcohols } \\
\hline \multicolumn{5}{|l|}{ Aliphatic alcohols } \\
\hline 1-pentanol & $t^{b}$ & $\mathrm{t}^{\mathrm{b}}$ & $t^{b}$ & $6.62^{\mathrm{a}}$ \\
\hline 2,3-butanediol & $t^{b}$ & $\mathrm{t}^{\mathrm{b}}$ & $3.10^{\mathrm{b}}$ & $12.88^{\mathrm{a}}$ \\
\hline 1-octen-3-ol & $1.58^{\mathrm{b}}$ & $2.23^{\mathrm{b}}$ & $2.59^{\mathrm{b}}$ & $7.80^{\mathrm{a}}$ \\
\hline 2-octen-1-ol & $t^{b}$ & $t^{b}$ & $t^{b}$ & $2.25^{\mathrm{a}}$ \\
\hline 2-nonen-1-ol & $t^{d}$ & $3.43^{\mathrm{c}}$ & $6.75^{b}$ & $10.73^{\mathrm{a}}$ \\
\hline 2-decen-1-ol & $\mathrm{t}^{\mathrm{c}}$ & $0.86^{\mathrm{b}}$ & $2.71^{\mathrm{a}}$ & $2.27^{\mathrm{a}}$ \\
\hline \multicolumn{5}{|l|}{ Branched alcohols } \\
\hline 1-methoxy-2-propanol & $t^{c}$ & $t^{c}$ & $7.50^{\mathrm{b}}$ & $30.47^{\mathrm{a}}$ \\
\hline 3-methyl-3-buten-1-ol & 1.76 & 1.65 & 1.13 & 0.40 \\
\hline 3-methyl-1-butanol & $t^{b}$ & $t^{b}$ & $10.26^{\mathrm{a}}$ & $9.46^{\mathrm{a}}$ \\
\hline 1-butoxy-2-propanol & $0.66^{\mathrm{b}}$ & $0.26^{\mathrm{c}}$ & $0.70^{\mathrm{b}}$ & $1.17^{\mathrm{a}}$ \\
\hline 2-ethyl-1-hexanol & $9.18^{\mathrm{b}}$ & $7.94^{\mathrm{b}}$ & $12.67^{\mathrm{a}}$ & $6.98^{\mathrm{b}}$ \\
\hline 2-butyl-1-octanol & $0.03^{\mathrm{d}}$ & $0.31^{\mathrm{c}}$ & $1.10^{\mathrm{a}}$ & $0.61^{b}$ \\
\hline 2-ethyl-1-decanol & $t^{b}$ & $0.25^{\mathrm{b}}$ & $1.32^{\mathrm{a}}$ & $1.08^{\mathrm{a}}$ \\
\hline \multicolumn{5}{|l|}{ Aldehydes } \\
\hline 3-methyl-butanal & $0.64^{\mathrm{c}}$ & $t^{c}$ & $1.63^{\mathrm{b}}$ & $4.02^{\mathrm{a}}$ \\
\hline hexanal & $t^{c}$ & $1.04^{\mathrm{c}}$ & $5.23^{\mathrm{b}}$ & $8.08^{\mathrm{a}}$ \\
\hline heptanal & $\mathrm{t}^{\mathrm{b}}$ & $t^{b}$ & $t^{b}$ & $1.63^{\mathrm{a}}$ \\
\hline \multicolumn{5}{|l|}{ Ketones } \\
\hline 2-pentanone & 2.05 & 0.92 & 1.56 & 1.48 \\
\hline 3-hydroxy-2-butanone & $4.31^{\mathrm{b}}$ & $4.60^{\mathrm{b}}$ & $18.75^{\mathrm{b}}$ & $67.10^{\mathrm{a}}$ \\
\hline 4-hydroxy-4-methyl-2-pentanone & $t^{b}$ & $t^{b}$ & $t^{b}$ & $0.91^{\mathrm{a}}$ \\
\hline 4-methyl-2-hexanone & 2.51 & 2.81 & 3.05 & 3.12 \\
\hline 2-methyl-3-octanone & $1.98^{\mathrm{b}}$ & $0.85^{\mathrm{d}}$ & $1.39^{\mathrm{c}}$ & $2.99^{\mathrm{a}}$ \\
\hline \multicolumn{5}{|l|}{ Esters } \\
\hline methyl butanoate & $2.67^{\mathrm{a}}$ & $0.37^{\mathrm{b}}$ & $t^{b}$ & $t^{b}$ \\
\hline 3-methyl-1-butyl acetate & 1.44 & 0.39 & 1.62 & 2.62 \\
\hline methyl hexanoate & $0.89^{\mathrm{b}}$ & $0.31^{\mathrm{c}}$ & $0.91^{\mathrm{b}}$ & $1.60^{\mathrm{a}}$ \\
\hline 3-methylheptyl acetate & $0.78^{\mathrm{c}}$ & $1.63^{\mathrm{bc}}$ & $3.92^{\mathrm{a}}$ & $2.11^{\mathrm{b}}$ \\
\hline heptyl butanoate & $1.24^{\mathrm{b}}$ & $0.58^{\mathrm{c}}$ & $1.85^{\mathrm{a}}$ & $2.15^{\mathrm{a}}$ \\
\hline \multicolumn{5}{|l|}{ Carboxylic acid } \\
\hline butanoic acid & $2.64^{\mathrm{a}}$ & $1.93^{\mathrm{a}}$ & $0.43^{\mathrm{b}}$ & $t^{b}$ \\
\hline \multicolumn{5}{|l|}{ N-containing compounds } \\
\hline ethylamine & $\mathrm{t}$ & 0.53 & 1.66 & 2.19 \\
\hline 4-amino-1-butanol & $\mathrm{t}$ & $\mathrm{t}$ & 0.81 & 0.21 \\
\hline pyridine & $\mathrm{t}$ & 0.25 & 0.77 & 0.90 \\
\hline \multicolumn{5}{|l|}{ S-containing compounds } \\
\hline 1-propanethiol & $t^{b}$ & $t^{b}$ & $0.60^{\mathrm{b}}$ & $4.58^{\mathrm{a}}$ \\
\hline acetyl mercaptan & $0.07^{\mathrm{c}}$ & $1.56^{\mathrm{c}}$ & $15.54^{\mathrm{b}}$ & $22.48^{\mathrm{a}}$ \\
\hline \multicolumn{5}{|l|}{ Furans } \\
\hline tetrahydro-2,2,4,4-tetramethyl-furan & $0.81^{\mathrm{c}}$ & $0.66^{\mathrm{c}}$ & $2.10^{\mathrm{b}}$ & $2.81^{\mathrm{a}}$ \\
\hline dibenzofuran & $0.18^{\mathrm{a}}$ & $0.12^{\mathrm{b}}$ & $0.07^{\mathrm{c}}$ & $0.16^{\mathrm{ab}}$ \\
\hline
\end{tabular}

$\overline{\mathrm{a}-\mathrm{d}}$ Means with a same superscript within a same row are not different $(p>0.05) .{ }^{1)} \mathrm{t}=$ trace amount. 
1-phenyl-1-butanone.

\section{Other Volatile Compounds}

Esters, such as methyl butanoate, methyl hexanoate and 3 -methyl-1-butyl acetate decreased $(p<0.05)$ in concentration when exposed to the heat treatment, whereas, concentration of 2-ethylhexyl-2-propenoate increased with heating. Also, pentyl hexanoate, 3-methylbutyl butanoate and 3-methylheptyl acetate had higher concentrations $(p<0.05)$ in the two-phase fiber, whereas heptyl butanoate, 2-butoxyethyl acetate, 2-ethylhexyl-2-propenoate and 2ethylhexyl butyrate had higher concentrations $(p<0.05)$ when the three phase fiber was used. For carboxylic acids, butanoic acid and oleic acid were affected $(p<0.05)$ by heat treatment. 2-methyl-2-ethyl-propanoic acid and oleic acid had higher concentrations $(p<0.05)$ when extracted by the three-phase fiber as compared to when extracted with the two phase fiber. For nitrogen-compounds, cyclobutylamine and 4-amino-1-butanol, and ethylamine were affected $(p<0.05)$ by heat treatment and fiber type, respectively $(p<0.05)$. Sulfur-compounds such as methanethiol, acetyl mercaptan, 2-methyl-3-pentanethiol, dimethyl trisulfide and dimethyl tetrasulfide were affected $(p<0.05)$ by heat treatment, but were not affected $(p>0.05)$ by fiber type with the exception of acetyl mercaptan and 2-methyl-3-pentanethiol. These compounds increased $(p<0.05)$ with heat treatment, with the exception of acetyl mercaptan. All furans detected from loins decreased $(p<0.05)$ when treated with heat, with the exception of 2-pentyl-furan. 2-pentyl-furan also had the highest concentration $(p<0.05)$ of all furans in pre-heated loins, which is in agreement with results reported by Poligne et al. (2002). They reported that the concentration of 2-pentyl-furan in pork bellies increased due to cooking.

\section{Effect of Storage Time on Volatile Compounds}

Forty-two volatile compounds were identified from porcine LD muscle during storage, including hydrocarbons (8), alcohols (13), aldehydes (3), ketones (5), esters

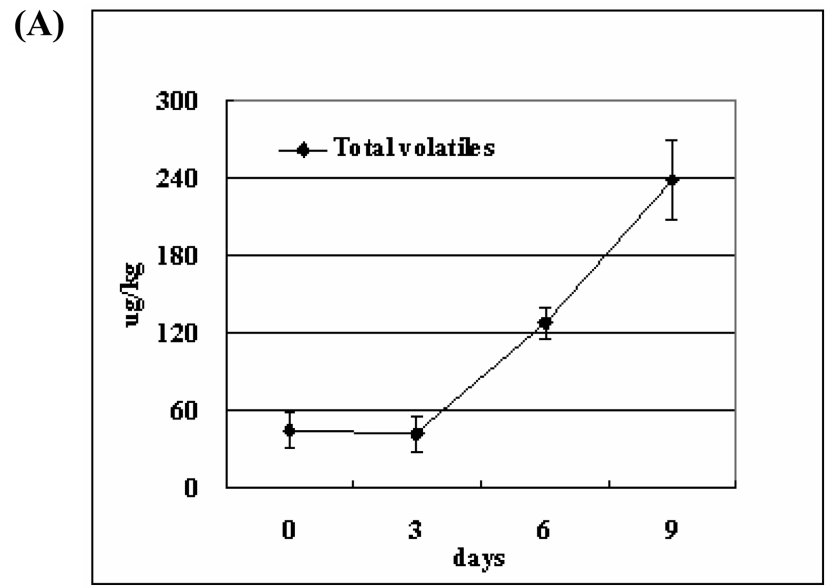

(B)

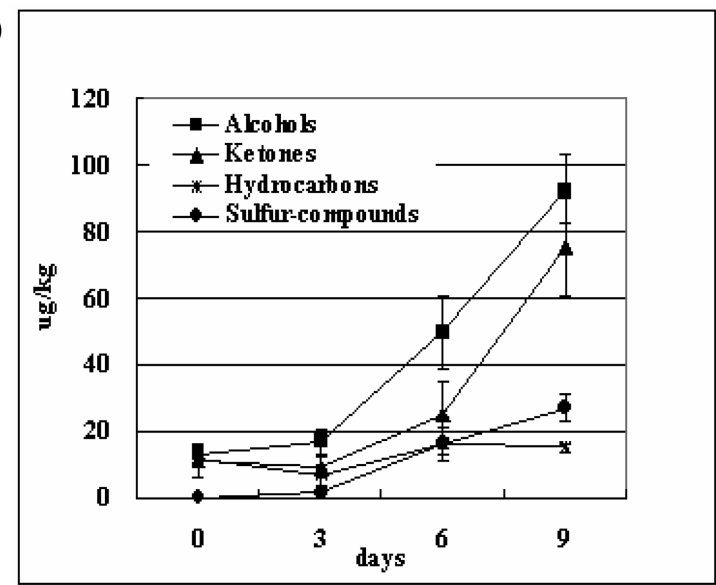

(C)

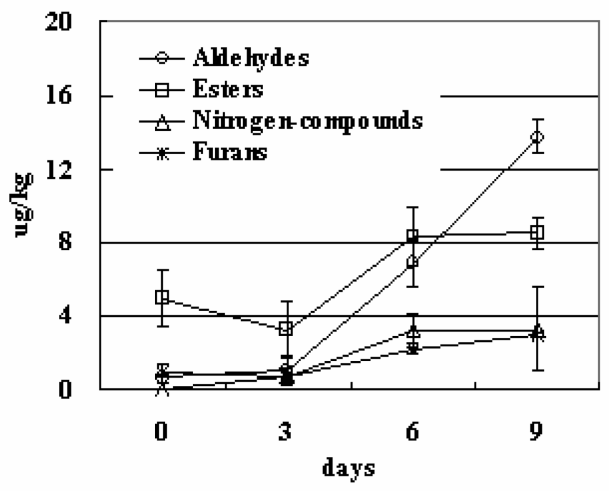

Fig. 1. Changes of various volatile compounds extracted from loins during storage time at $4^{\circ} \mathrm{C}$. (A): total volatile compounds, $(\mathrm{B})$ : alcohols, ketones, hydrocarbons and sulfur-containing compounds, (C): aldehydes, esters, nitrogen-containing compounds and furans. 
(5), carboxylic acid (1), nitrogen compounds (3), sulfur compounds (2) and furans (2), when the two-phase fiber was used for extraction (Table 5). Total volatile compound concentration increased $(p<0.05)$ from 44.9 to 239 $\mu \mathrm{g} / \mathrm{kg}$ over $9 \mathrm{~d}$ of storage. (Fig. 1-A). Alcohols and ketones were the chemical groups that were most abundant at the final storage time, their concentrations increased $(p<0.05)$ from 13.21 to $92.78 \mu \mathrm{g} / \mathrm{kg}$ and from 10.85 to $75.61 \mu \mathrm{g} /$ $\mathrm{kg}$, respectively. 1-methoxy-2-propanol and 3-hydroxy-2butanone had the greatest increase in concentration over the storage time. In general, refrigeration of fresh meat caused a small increase in the concentration of aldehydes that are generally derived from lipid oxidation and a large increase in methyl alcohols and ketones that are usually generated from branched chain amino acids and pyruvate catabolism (Estevez et al., 2003).

Most alcoholic compounds that were identified from pork loins increased $(p<0.05)$ over storage time except for 3-methyl-3-buten-1-ol. Increasing in concentrations of any alcoholic compounds may be partially due to the catabolism of glutamic acid, which produces alcohols via $\alpha$-keto acids (Ardo, 2006). Especially, 1-methoxy-2-propanol, 2,3-butanediol, 2-nonen-1-ol and 3-methyl-1butanol concentrations increased dramatically $(p<0.05)$, resulting in increases in total aliphatic and branched alcohols. For instance, 2,3-butanediol and 3-methyl-1-butanol are generated from pyruvate and leucine or isoleucine metabolism via Strecker degradation and have flavor notes such as buttery and fruity (Montel et al., 1998; Urbach, 1995). Also, 2,3-butanediol might be probably generated from catabolism of aspartic acid, which produces oxaloacetate to be catabolised into diacetyl, acetoin and 2,3-butanediol (Ardo, 2006). It also probably contributes to the aroma of refrigerated meat since it has a low threshold (0.002 ppm) value (Montel et al., 1998). 1-pentanol, 2,3-butanediol, 2-octen-1-ol, 2-nonen-1-ol, 2-decen1-ol, 1-methoxy-2-propanol, 3-methyl-1-butanol and 2ethyl-1-decanol were not detected at $0 \mathrm{~d}$ of storage, but were detected as storage time increased. The concentrations of aldehydes, ketones and esters increased $(p<0.05)$ over storage time, but that of methyl butanoate decreased $(p<0.05)$ with increased storage time. Among all volatile compounds identified from pork loins, 3-hydroxy-2butanone had the highest increase over time and caused a large increase in total ketones concentration. Estevez et al. (2003) found that 3-hydroxy-2-butanone in pork loins was one of the volatile compounds that showed remarkable increases during storage at $4^{\circ} \mathrm{C}$ for $10 \mathrm{~d}$, and announced 3-hydroxy-2-butanone as an meat aging indica- tor. Hexanal also increased $(p<0.05)$ during storage time, indicating increased oxidation over the storage time. According to the result of Park et al. (2008) who studied effects of packaging type on oxidative volatile compounds of pork meats, vacuum packaging inhibited the production of oxidative volatile compounds as compared to aerobic packaging, especially in alcohols.

\section{Conclusion}

Heating significantly changed the volatile flavor composition of pork M. longissimus dorsi, as well as induced the formation of volatile compounds, especially hexanal, 1-octen-3-ol and 2,3-octanedione. 2-ethyl-1-hexanol was significantly decreased and might be converted into ester (2-ethylhexyl-2-propenoate) by heating. Two-phase fibers (Carboxen-PDMS) appeared to be more suitable for measuring low molecular weight compounds, whereas threephase fibers (DVB-Carboxen on PDMS) appeared to be more suitable for measuring high molecular weight compounds. Increasing storage time led to the formation of various volatile compounds from most chemical groups, especially alcohols (1-methoxy-2-propanol, 2,3-butanediol) and ketones (3-hydroxy-2-butanone). Overall, heating and refrigerated storage of pork M. longissimus dorsi increased volatile compounds derived from lipid oxidation and amino acid catabolism, respectively.

\section{Acknowledgements}

This study was supported by the Brain Korea 21 program (Center for the control of animal hazards using biotechnology, Chonnam National University) and partially supported by Biotechnology Research Institute, Chonnam National University, Gwangju, Korea.

\section{References}

1. Ahn, D. U., Jo, C., and Olson, D. G. (1999) Volatile profiles of raw and cooked turkey thigh as affected by purge temperature and holding time before purge. J. Food Sci. 64, 230233.

2. Ardo, Y. (2006) Flavour formation by amino acid catabolism. Biotechnol. Adv. 24, 238-242.

3. Beltran, E., Pla, R., Yuste, J., and Mor-Mur, M. (2003) Lipid oxidation of pressurized and cooked chicken: role of sodium chloride and mechanical processing on TBARS and hexanal values. Meat Sci. 64, 19-25.

4. Brunton, N. P., Cronin, D. A., and Monahan, F. J. (2001) The effects of temperature and pressure on the performance of 
Carboxen/PDMS fibres during solid phase microextraction (SPME) of headspace volatiles from cooked and raw turkey breast. Flavour Fragr. J. 16, 294-302.

5. Estevez, M., Morcuende, D., Ventanas, S., and Cava, R. (2003) Analysis of volatiles in meat from Iberian pigs and leas pigs after refrigeration and cooking by using SPME-GCMS. J. Agr. Food Chem. 51, 3429-3435.

6. Garcia-Esteban, M., Ansorena, D., Astiasaran, I., and Ruiz, J. (2004) Study of the effect of different fiber coatings and extraction conditions on dry cured ham volatile compounds extracted by solid-phase microextraction (SPME). Talanta 64, 458-466.

7. Gianelli, M. P., Flores, M., and Toldra, F. (2002) Optimisation of solid phase microextraction (SPME) for the analysis of volatile compounds in dry-cured ham. J. Sci. Food Agr. 82, 1703-1709.

8. Machiels, D. and Istasse, L. (2003) Evaluation of two commercial solid-phase microextraction fibres for the analysis of target aroma compounds in cooked beef meat. Talanta 61, 529-537.

9. Montel, M. C., Masson, F., and Talon, R. (1998) Bacterial role in flavour development. Meat Sci. 49, S111-S123.

10. Morcuende, D., Estevez, M., Ruiz, J., and Cava, R. (2003) Oxidative and lipolytic deterioration of different muscles from free-range reared Iberian pigs under refrigerated storage. Meat Sci. 65, 1157-1164.

11. Mottram, D. S. (1998) Flavour formation in meat and meat products: a review. Food Chem. 62, 415-424.

12. Park, S. Y., Kim, Y. J., Lee, H. C., Yoo, S. S., Shim, J. H., and Chin, K. B. (2008). Effects of pork meat cut and packaging type on lipid oxidation and oxidative products during refrigerated storage $\left(8^{\circ} \mathrm{C}\right)$. J. Food Sci. 73, C127-C134.

13. Poligne, I., Collignan, A., and Trystram, G. (2002) Effects of salting, drying, cooking, and smoking operations on volatile compound formation and color patterns in pork. J. Food Sci. 67, 2976-2986.

14. Ruiz, J., Cava, R., Ventanas, J., and Jensen, M. T. (1998) Headspace solid phase microextraction for the analysis of volatiles in a meat product: dry-cured Iberian ham. J. Agr. Food Chem. 46, 4688-4694.

15. Sanches-Silva, A., Rodriguez-Bernaldo de Quiros, A., LopezHernandez, J., and Paseiro-Losada, P. (2004) Determination of hexanal as indicator of the lipidic oxidation state in potato crisps using gas chromatography and high-performance liquid chromatography. J. Chromatogr. A 1046, 75-81.

16. Stephen Elmore, J., Mottram, D. S., and Hierro, E. (2000) Two-fibre solid-phase microextraction combined with gas chromatography-mass spectrometry for the analysis of volatile aroma compounds in cooked pork. J. Chromatogr. A 905, 233-240.

17. Urbach, G. (1995) Contribution of lactic acid bacteria to flavour compound formation in dairy products. Int. Dairy J. $\mathbf{5}$, 877-903.

(Received 2009.7.29/Revised 2009.10.5/Accepted 2009.10.5) 